\title{
Training-Aided Channel Estimation and Equalization in SDM Systems with MISO Pre-convergence under Strong Coupling
}

\author{
Christian Sánchez ${ }^{(1)}$, Filipe Ferreira(1), Jinlong Wei(2), Stylianos Sygletos ${ }^{(1)}$, Andrew Ellis ${ }^{(1)}$ \\ (1) Aston Institute of Photonic Technologies (AIPT), Aston University, Birmingham, B4 7ET, UK, \\ c.sanchez-costa@aston.ac.uk \\ (2) Huawei Technologies Duesseldorf GmbH, European Research Center, Riesstrasse 25, 80992 \\ München, Germany
}

\begin{abstract}
A$ simple DSP scheme receiver is proposed to circumvent laser frequency-offset effects in $D A-C E$ based SDM systems using a MISO CMA pre-convergence stage. Numerical results demonstrate a successful operation for 12-mode fiber transmission under MDL, using QPSK and 16QAM mapping.
\end{abstract}

\section{Introduction}

The exploitation of the spatial domain for optical parallel transmission in coherent optical communication systems is foreseen as the only solution to keep up with the demanded information rates in the long-term ${ }^{1}$. Different variants of links to take advantage of the spatial dimension can be used ${ }^{2}$, presenting ranging levels of signal mixing/coupling along the link, and, thus, needing different digital-signal processing (DSP) algorithms at the receiver. This work focus on few-mode fibre (FMF)-based systems, assuming strong coupling between signals and making necessary multiple-input multiple-output (MIMO) equalization.

In single-mode fibre (SMF) based links, the conventional solution relies on non-data aided transmission and $2 \times 2$ MIMO equalization for polarization de-multiplexing using constantmodulus algorithm (CMA) with least-mean squares (LMS) filter updating. One of the most prominent advantages of CMA is its insensitiveness to the frequency offset (FO) and the phase noise present in optical coherent receivers, allowing the independent operation of the channel equalization and the carrier recovery stages. Moreover, signal propagation conditions on SMF have made possible elegant ways to overcome problems derived from its inherent illconvergence ${ }^{3}$. As an example, in ${ }^{4} \mathrm{CMA}$ is used in a first stage (pre-convergence) for the determination of an initial estimate of the FO. In contrast, solutions based on data-aided transmission and channel estimation (DA-CE) and subsequent equalization have received less attention, though it offers potential benefits ${ }^{5,6}$.

Signal propagation effects in SDM-FMF systems challenges the conventionally adopted solutions in SMF based systems due to the reception of higher number of signals and FMF signal propagation impairments. A conventional $\mathrm{NxN}$ CMA equalizer might converge to an illconditioned solution and get the same signal at more than one equalizer output, problem which is aggravated by mode-dependent loss (MDL). In this scenario, authors have opted conventionally for the data-aided LMS algorithm, making necessary the transmission of long sequences to train the equalizer and finely compensate for $\mathrm{FO}$ and laser phase noise effects using a feedbackloop in order to ensure a correct equalization operation. Recent work has explored Stokes space in SDM-FMF systems ${ }^{7}$ with the aim of performing equalization in the frequency domain (FD) without being affected by FO effects.

In this paper we propose a simple solution aimed to benefit of the advantages of well-stablished algorithms in SMF-based systems. A preconvergence multiple-input single-output (MISO) equalization stage using CMA gets only one equalized signal from which a reliable $\mathrm{FO}$ estimation can be obtained. Once the FO is compensated for in the whole set of received signals, we use the transmitted training sequences to estimate the $12 \times 12$ channel impulse responses (CIR), which are employed to initialize the MIMO channel-tracking equalizer. Our proposal avoids the channel estimation and equalization sensitivity to phase shifts due to FO as well as ill-convergence of CMA under the presence of MDL. Moreover, since it relies on the successful recovery of only one signal and DA$\mathrm{CE}$ for equalization initialization, it can potentially reduce the length of the sequence used for channel equalizer acquisition.

\section{SDM system model}

The simulated SDM system is shown in Fig. 1(a). Twelve 28GBd signals are transmitted, one per orthogonal polarization of a 6-mode FMF (LP01, $\left.L P_{11 a}, L P_{11 b}, L P_{02}, L P_{21 a}, L P_{21 b}\right)$, achieving a total line rate of $672 \mathrm{~Gb} / \mathrm{s}(1344 \mathrm{~Gb} / \mathrm{s})$ with QPSK (16QAM). Together with the information data, a preamble is transmitted consisting of constant sequences, used at the receiver for time synchronization and channel estimation (Fig. $1(b))$. Each of the signals drives an optical IQ modulator which modulates the output of a single 


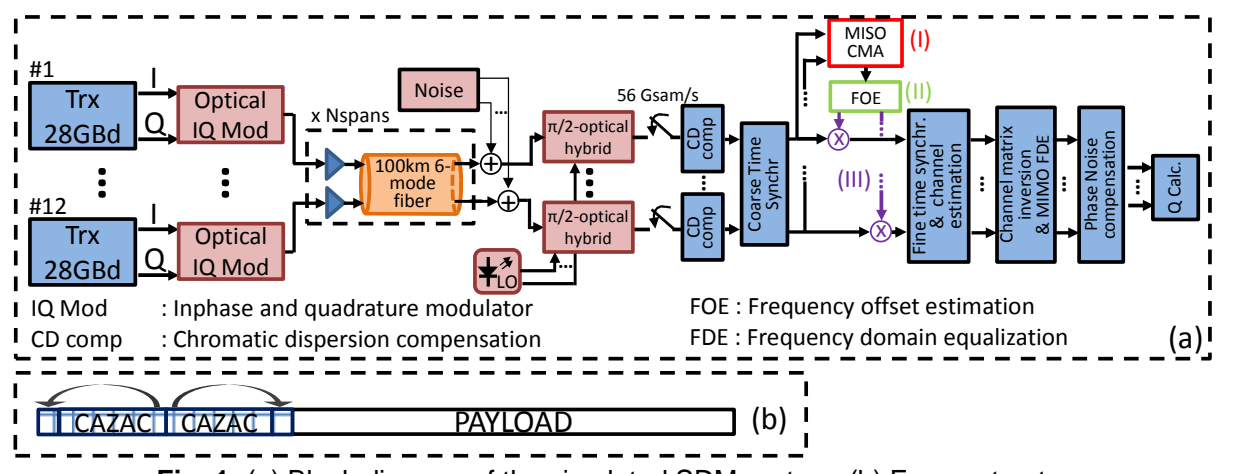

Fig. 1: (a) Block diagram of the simulated SDM system. (b) Frame structure.

$100 \mathrm{kHz}$ linewidth laser, and then they are combined and launched into the FMF link, composed of $\mathrm{N}_{\text {spans }}$ of $100 \mathrm{~km}$ amplitude zero autocorrelation (CAZAC) length each. The fibre is assumed to be linear and lossless. The fibre is divided into sections of $100 \mathrm{~m}$, and coupling between signals is modelled by random unitary matrixes. The differential-mode delay is set to $25 \mathrm{ps} / \mathrm{km}$. MDL is introduced through unequal amplifier gains for the six modes, extracted from a uniform distribution determined by the desired MDL. After propagation, optical signals are noise loaded to the wanted OSNR. Square-root raised cosine filters with a roll-off factor of 0.4 are used at the transmitter and the receiver sides.

After intradyne detection with a $100 \mathrm{kHz}$ linewidth laser, the baseband electrical signals are sampled at $56 \mathrm{Gsam} / \mathrm{s}$, yielding twelve digital signals with 2samples/symbol. The first stage of the DSP is the compensation of chromatic dispersion in the frequency domain. The beginning of the frame is detected using the Schmidl \& Cox autocorrelation metric. Next, MISO pre-equalization in the frequency domain with CMA filter updating is applied ((I) in Fig.1(a)), with which one of the transmitted signals is recovered. Once the equalizer has converged, we use the $4^{\text {th }}$-power FO estimation algorithm (II) (or a finer one, such as that in ${ }^{8}$ ), and compensate for the FO all of the 12 received signals (III). Without significant FO effects, fine time synchronization and $\mathrm{CE}$ can be performed by cross-correlating the received signals with the training sequences. Then the $12 \times 12$ estimated CIRs are converted into the FD. The initial MIMO FD equalizer is calculated by inverting the channel matrix, after which CMA is again used for channel tracking. Phase noise compensation is performed, and, finally, the $\mathrm{Q}^{2}$-factor is calculated. For the calculation of the $\mathrm{Q}^{2}$-factor we have used a corrected version of the non-data aided error-vector magnitude ${ }^{9}$.

\section{DA-CE and Equalization under FO}

The main difficulty of DA-CE transmission systems comes from the interdependence of time and frequency synchronization with channel effects compensation. Yet, little progress ${ }^{10}$ has been achieved on overcoming its high sensitivity to the FO caused by the frequency mismatch of the transmitter and receiver lasers. In Fig.2 we show the $\mathrm{Q}^{2}$-factor obtained as a function of the $\mathrm{FO}$ in a DA-CE with $\mathrm{N}_{\text {spans }}=3$, OSNR $=21 \mathrm{~dB}$ and QPSK mapping. To achieve a BER of $10^{-3}$, a $Q^{2}$ factor equal to $9.8 \mathrm{~dB}$ is needed. As observed in Fig. 2, even with a FO as small as $8 \mathrm{MHz}$ the $\mathrm{Q}^{2}$ factor falls below $9.8 \mathrm{~dB}$. Since coarse frequency estimators have a tuning range in the order of $\sim \mathrm{GHz}$, their employment does not guarantee a proper FO compensation before channel estimation and a finer estimation is needed. Since fine FO estimation algorithms need channel distorted-less symbols, we find ourselves with a causality dilemma in DA-CE FMF-SDM systems.

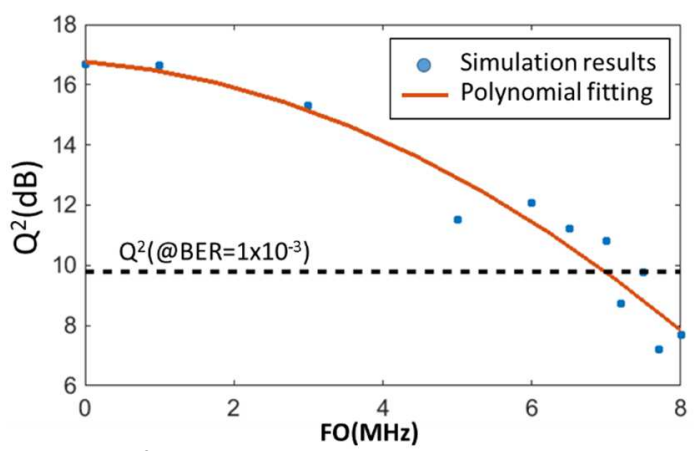

Fig. 2: $Q^{2}$-factor in function of the $F O$ in conventional DA-CE system (Nspans $=3$, OSNR=21dB).

\section{Numerical results}

In this section we numerically evaluate the proposed receiver DSP configuration to overcome the mentioned limitations in FMF-SDM systems. The transmitted signal (1 frame) is composed of a total of $2^{17}$ symbols, of which 2304 are overhead. The lasers frequency mismatch is set to $1 \mathrm{GHz}$, a value unacceptable for correct

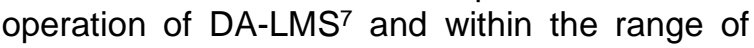
coarse FO estimators. In Fig.3 we show the $\mathrm{Q}^{2}$ factor obtained after 3 spans of $100 \mathrm{~km}$ FMF fibre 
and $\mathrm{MDL}=0,1$ and $2 \mathrm{~dB}$ using QPSK. The theoretical $\mathrm{Q}^{2}$-curve under additive white Gaussian noise for QPSK is also shown for reference.

We can observe in Fig. 3 some roughly penalty compared to the theoretical curve, which is between 1-2dB for OSNR values between 15 and $20 \mathrm{~dB}$. In order to obtain an average BER of $10^{-3}$,

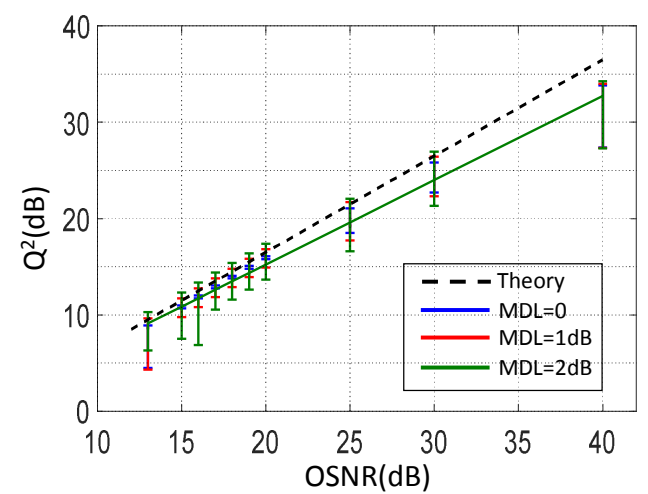

Fig. 3: $Q^{2}$-factor in function of OSNR and different values of $\mathrm{MDL}$ for $300 \mathrm{~km}$. Vertical bars indicate the minimum $Q^{2}$-factor from the 12 received signals over the 100 realizations. Solid line: linear fitting.

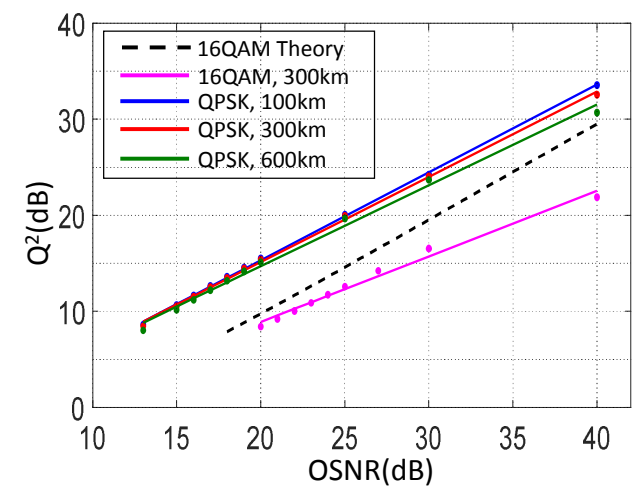

Fig. $4 Q^{2}$-factor in function of OSNR, distances $=100 \mathrm{~km}, 300 \mathrm{~km}, 600 \mathrm{~km}, \mathrm{MDL}=1 \mathrm{~dB}$. (Dots) Results averaged over 40 realizations. Solid lines: linear fitting

an OSNR around $15 \mathrm{~dB}$ is required, which is in accordance with previously reported values ${ }^{5}$. The average performance, favoured by the strong coupling in the FMF propagation model, is little affected by MDL, which effects are more observable in the $\mathrm{Q}^{2}$-factor deviation represented by the vertical bars. No ill-conditioned solution was detected thanks to the DA-CE initialization of the tracking equalizer despite of MDL. In Fig. 4 we plot the $\mathrm{Q}^{2}$-factor in function of the OSNR for $N_{\text {spans }}=1,3$ and 6, using QPSK, and for $N_{\text {spans }}=3$ using 16QAM, and MDL=1dB. We can observe a negligible penalty when increasing the distance for QPSK. For 16QAM and $\mathrm{N}_{\text {spans }}=3$, the penalty compared to the theory is around $2 \mathrm{~dB}$ for OSNR values between 20 and $25 \mathrm{~dB}$, being necessary an OSNR of around $21 \mathrm{~dB}$ to achieve an average BER of $10^{-3}$.

\section{Conclusions}

In this work we have proposed a receiver DSP configuration which attains the main advantages of well-known receiver DSP algorithms proposed for SMF and DA-CE -based systems. FMF propagation, phase noise and $\mathrm{FO}$ effects are successfully overcome, while MDL does not induce CMA ill-convergence problems thanks to the DA-CE initialization. The results also show its compatibility with 16QAM mapping. MISO convergence and DA-CE favour our proposal in terms of channel equalizer acquisition compared to others, though further work is still needed to improve the convergence time beyond conventional stochastic-gradient CMA based approaches for higher distances and modulation formats.

\section{Acknowledgements}

This work has been partially supported by the European Union (Grants 659950-INVENTION, 619732-INSPACE, 654809-HSPACE, 623515CEEOALAN), and by the EPSRC (Grant EP/L000091/1-PEACE).

http://doi.org/10.17036/researchdata.aston.ac.uk.000 $\underline{00295}$

\section{References}

[1] P. J. Winzer, "Spatial multiplexing in fiber optics: the $10 \mathrm{X}$ scaling of metro/core capacities," Bell Labs Tech. J., Vol. 19, p. 22 (2014).

[2] D. J. Richardson et al., "Space-division multiplexing in optical fibres," Nat. Photonics. Vol. 7, p. 354 (2013).

[3] L. Liu et al., "Initial tap setup of constant modulus algorithm for polarization de-multiplexing in optical coherent receivers," Proc. OFC, OMT2, San Diego (2009).

[4] P. J. Winzer et al., "Spectrally efficient long-haul optical networking using 112-Gb/s polarization-multiplexed 16QAM,” J. Lightwave Technol., Vol. 28, no. 4, p. 547 (2010).

[5] F. Pittalà et al., "Training-aided frequency-domain channel estimation and equalization for single-carrier coherent optical transmission systems," J. Lightwave Technol., Vol. 32, No. 24, p. 4849 (2014).

[6] M. Kuschnerov et al., "Data-aided versus blind singlecarrier coherent receivers," IEEE Photonics J., Vol. 2, No. 3, p. 387 (2010).

[7] F. J. V. Caballero et al., "Novel equalization techniques for space division multiplexing based on Stokes space update rule," Photonics, Vol. 4, no. 1 (2017).

[8] M. Selmi et al.,"Accurate digital frequency offset estimator for coherent PolMux QAM transmission systems," Proc. Eur. Conf. Opt. Commun., 2009, pp. 1.

[9] Hisham A. et al., "Error vector magnitude to SNR conversion for nondate-aided receivers," IEEE Trans. Wireless Commun., Vol. 8, No. 5, p. 2694 (2009).

[10]F. Pittalà et al., "Joint frame synchronization and frequency offset estimation in coherent optical 
transmission systems," Proc. ECOC, Mo4D4, London (2013). 